\title{
Procoagulant reactivity to laboratory acute mental stress in Africans and Caucasians, and its relation to depressive symptoms: The SABPA Study
}

Running title: Procoagulant stress reactivity in Africans

\author{
1,2 Roland von Känel, MD \\ ${ }^{3,4}$ Mark Hamer, PhD \\ ${ }^{4}$ Nico T. Malan, DSc \\ ${ }^{4}$ Kobus Scheepers, BSc Hons \\ ${ }^{5}$ Muriel Meiring, $\mathrm{PhD}, \mathrm{MBA}$ \\ ${ }^{4}$ Leoné Malan, RN, PhD
}
${ }^{1}$ Division of Psychosomatic Medicine, Department of General Internal Medicine, Inselspital, Bern University Hospital and University of Bern, Switzerland
${ }^{2}$ Department of Clinical Research, University of Bern, Switzerland
${ }^{3}$ Department of Epidemiology and Public Health, University College London, UK
${ }^{4}$ Hypertension in Africa Research Team (HART), Faculty of Health Sciences North West University, Potchefstroom, South Africa.
${ }^{5}$ Department of Haematology and Cell Biology, University of the Free State, Bloemfontein, South Africa

Address for correspondence:

Roland von Känel, MD

Professor of Psychosomatic and Psychosocial Medicine

Department of General Internal Medicine

Inselspital, Bern University Hospital

CH-3010 Bern, Switzerland

Tel.: +41 (0) 3163220 19; fax: +41 (0) 313821184

E-mail: roland.vonkaenel@insel.ch 


\section{SUMMARY}

The risk of cardiovascular disease is dramatically increasing in Africans (black). The prothrombotic stress response contributes to atherothrombotic disease and is modulated by depressive symptom. We examined coagulation reactivity to acute mental stress and its relation to psychological well-being in Africans relative to Caucasians (white). 102 African and 165 Caucasian school teachers underwent the Stroop Color-Word Conflict test. Circulating levels of von Willebrand factor (VWF) antigen, fibrinogen, and D-dimer were measured before and after the Stroop. Cardiovascular reactivity measures were also obtained. All participants completed the Patient Health Questionnaire-9 and the General Health Questionnaire-28 for the assessment of depressive symptoms and total psychological distress, respectively. After controlling for covariates, resting levels of VWF, fibrinogen, and D-dimer were higher in Africans than in Caucasians (all p-values $\leq 0.006$ ). Depressive symptoms and psychological distress were not significantly associated with resting coagulation measures. Stress reactivity in VWF $(\mathrm{p}<0.001)$ and fibrinogen $(\mathrm{p}=0.016)$, but not in $\mathrm{D}$-dimer $(\mathrm{p}=0.27)$, were decreased in Africans relative to Caucasians with Africans showing greater reactivity of total peripheral resistance $(\mathrm{p}=0.017)$. Depressive symptoms, but not general psychological distress, were associated with greater VWF increase $(\mathrm{p}=0.029)$ and greater fibrinogen decrease $(\mathrm{p}=0.030)$ in Africans relative to Caucasians. In conclusion, Africans showed greater hypercoagulability at rest but diminished procoagulant reactivity to acute mental stress when compared with Caucasians. Ethnic differences in the vascular adrenergic stress response might partially explain this finding. Depressive symptoms were associated with exaggerated VWF reactivity in Africans relative to Caucasians. The clinical implications of these findings for Africans need further study.

Keywords: Cardiovascular disease, coagulation, depression, ethnicity, psychological stress 


\section{INTRODUCTION}

Enhanced coagulation, impaired fibrinolysis, endothelial activation, and hyperactive platelets play an important role in the development of atherogenesis, atherothrombosis, and acute coronary syndromes (ACS) (1). During acute mental stress, healthy individuals show a prothrombotic state that is viewed as an adaptive fight-flight response protecting the organism from excessive bleeding should injury occur (2, 3; for review). Fibrinogen, von Willebrand factor antigen (VWF:Ag) and D-Dimer, the latter indicating fibrin turnover, are particularly responsive to acute mental stress $(4,5)$. Excessive stress procoagulant changes might potentially increase the risk of incident cardiovascular disease (CVD) and recurrent CVD events (3). For instance, stress-induced fibrinogen increase was associated with carotid artery stiffness (6) and predicted an increase in ambulatory systolic blood pressure (SBP) over three years in healthy individuals (7). Owing to impaired endothelial anticoagulant function, patients with atherothrombotic diseases showed greater platelet activation (8) and D-dimer increase (9) than controls free of CVD. Moreover, patients with emotional triggers like depressive feelings in the two hours before ACS onset, showed greater platelet aggregation during laboratory mental stress than patients reporting non-emotional triggers (10). Depressive symptoms have also been associated with stress-induced elevation in platelet reactivity (11) and D-dimer (12) in elderly subjects, thereby supporting the notion that hypercoagulability is one mechanism linking depression with an increased CVD risk (13).

Potential ethnical differences in the acute procoagulant response to stress and their relation to depressive symptoms have not previously been explored. Such biobehavioral research seems important for urbanized Africans in whom a concerning increase in CVD can be observed, much of it being a consequence of their transition from a traditional African to a modernized "Western" lifestyle (14). For instance, in Africans, the CVD risk predicted by fibrinogen becomes stronger with an increasing degree of urbanization (15). Moreover, subclinical atherosclerosis, as measured by increased carotid intima media thickness (CIMT), 
was greater in Africans than in Caucasians (16) as well as in those with more severe depressive symptoms of both these ethnicities (17).

The autonomic nervous system and hypothalamic pituitary adrenal axis are the two major physiologic stress response systems in humans. As cortisol peaks between 15 and 30 minutes after stress onset, prothrombotic changes within a few minutes of acute stress are largely governed by the sympathetic nervous system through release of catecholamines and adrenergic receptor stimulation $(18,19)$. Stress-hormone mediated stimulation of vascular beta2-adrenergic receptors releases VWF from endothelial cells (19-21). Stress-induced increases in D-dimer and in alpha-receptor activating norepinephrine are correlated with each other (5), but beta-adrenergic stimulation did not result in D-dimer increase (20). Compared to Caucasians, Africans show a greater alpha-adrenergic vascular response, which is reflected by a greater total peripheral resistance (TPR) (22). One specific hypothesis following from the above literature is that Africans would show greater D-dimer reactivity but lower VWF reactivity to acute stress than Caucasians. More severe depressive symptoms would hypothetically relate to greater procoagulant activity in both Africans and Caucasians.

The primary aim of this study was to investigate procoagulant reactivity between Africans and Caucasians through measurements of changes in circulating levels of VWF, fibrinogen and D-dimer to acute standardized laboratory stress. A secondary aim was to explore the association of depressive symptoms both continuously and categorically (i.e., based on a clinical cut-off level, with stress-induced coagulation changes. We further examined whether procoagulant reactivity would specifically relate to depressive mood or would rather be associated with elevated levels of general psychological distress.

\section{MATERIALS AND METHODS}

\section{Study participants and design}

The participants of this blood coagulation reactivity study were recruited as part of the 
Sympathetic Activity and Ambulatory Blood Pressure in Africans (SABPA) study conducted between February 2008 and May 2009. The study protocol was conducted in accordance with the Declaration of Helsinki (23) and was approved by the Ethics Review Board of the NorthWest University, Potchefstroom Campus (0003607S6). All participants provided written informed consent before participation. The SABPA study has a target population comparative design and recruited 409 teachers, aged 25-65 years, working in the Dr Kenneth Kaunda Education district in the North West Province, South Africa. This selection assured a homogenous sample from a similar socio-economic class. Exclusion criteria included pregnancy, lactation, and vaccination or blood donation within 3 months prior to participation. For purposes of the present study, we excluded participants with a history of atherothrombotic disease (myocardial infarction, $n=4$; stroke, $n=1)$, atrial fibrillation $(n=16)$, and HIV positive status $(\mathrm{n}=18)$, all of which are associated with hypercoagulability at rest and/or in response to acute stress $(10,24,25)$, as well as participants with an ear temperature $\geq 37.5^{\circ} \mathrm{C}(\mathrm{n}=3)$ because of a probable acute phase response that might increase coagulation factors like fibrinogen. Users of oral anticoagulants $(n=2)$, aspirin $(n=12)$, cortisone $(n=3)$, oral contraceptives $(n=21)$, antidepressants $(n=3)$, anxiolytics $(n=1)$, beta blockers $(n=4)$, and calcium antagonists $(\mathrm{n}=12)$ were also excluded, as all of these might affect resting coagulation activity and/or coagulation reactivity with acute mental stress and/or mood (21, 26-29). To allow full multivariate statistical analyses, subjects with we also excluded subjects who missed data for coagulation measures $(n=22)$, hemodynamic reactivity $(n=10)$, psychological questionnaires $(n=1)$, hemoglobin A1c $(\operatorname{HbA1c})(n=1)$, creatinine $(n=2)$, alcohol consumption $(n=1)$, and physical activity $(n=1)$. Finally, we excluded 4 participants because of excessively high D-dimer levels after stress ( $\geq 10^{\prime} 000 \mathrm{ng} / \mathrm{ml}$ ), yielding a final study sample of 267 subjects. Excluded participants $(\mathrm{n}=142)$ were significantly more often Africans, women, and antihypertensive drug users, and they also had significantly higher screening systolic blood pressure (SBP), HbA1c, and gamma glutamyl transferase ( $\gamma$-GT) levels than those included. 
The participants were admitted at 4:30 PM to the multi-bedroomed Metabolic Unit Research Facility of the North-West University on Monday through Thursday. All received a standardized dinner at 06:00 PM after which they completed psychological questionnaires. They had their last beverages (tea/coffee) and biscuits at 8:30 PM followed by recreational activities such as reading, watching television, or interacting socially. We also obtained information on demographic data, general health, including medication, and health behaviors. All participants went to bed at around 10:00 PM and were woken at 05:45 AM. After the completion of anthropometric and manual BP measurements, a registered nurse obtained fasting blood samples which were handled according to standardized procedures. A sterile winged infusion set was left in situ with a heparin block $(0.5 \mathrm{ml}$ of a Heparin SodiumFresenius $5000 \mathrm{IU} / \mathrm{ml}$ in 50ml normal saline solution; Fresenius Kabi, Port Elizabeth, South Africa) to prevent clotting. Thereafter, all participants underwent the laboratory stress.

\section{Acute laboratory stress testing}

Mental stress test: All participants underwent the Stroop Color-Word Conflict test for 1 minute. The Stroop is a standardized laboratory stressor showing reproducibility on cardiovascular reactivity (30). The Stroop requires identification of the ink color of the word rather than the name of the color spelled by the word, under time pressure. The participants received a monetary motivation reward in line with performance. Perceived stress triggered by the Stroop was rated on a Likert scale (1="not at all stressful", 7="very stressful").

Cardiovascular data collection: With participants in a semi-recumbent position a registered nurse took a 5-minute continuous measurement of resting cardiovascular parameters with the validated Finometer ${ }^{\circledR}$ device (Finapres Medical Systems, Amsterdam, the Netherlands) (31). The Finometer provides beat-by-beat BP and thus detects the full contour of the cardiovascular responses. The Finometer recorded the SBP, diastolic blood pressure (DBP) and heart rate (HR) and computed an integrated age dependent aortic flow 
curve from the surface area beneath the pressure/volume curve determining cardiac output (CO), stroke volume (SV), and total peripheral resistance (TPR) online (32); data were stored in the results files. Calculations of SV, CO, and TPR yield reliable measures $(33,34)$. We stabilized BP to resting state before participants underwent the Stroop. We obtained beat-tobeat $\mathrm{BP}$ responses throughout mental stress and the 5 min recovery phase. For analyses, we used the average of the last 2 minutes of the resting recordings and the averages of the last 15 seconds of the stressor recordings as well as of the recovery recordings at 1, 3, and 5 minutes after the Stroop had ended. Cardiovascular reactivity was calculated as the area under the curve (AUC) with respect to increase from rest (35) across these five time points for SBP, DBP, HR, CO, SV, and TPR.

Blood collection: Citrated blood samples were taken before the Stroop to determine resting levels of coagulation measures. At 10 minutes poststress, the infusion set was thoroughly flushed with 2-3 $\mathrm{ml}$ of saline and the first $2 \mathrm{ml}$ of blood were discarded before sampling was done for coagulation measures. Coagulation reactivity was calculated for each participant as the percentage change from the resting value.

\section{Cardiometabolic risk factors}

Body mass index: With participants in their underwear, we measured height and weight to the nearest $0.1 \mathrm{~cm}$ and $0.1 \mathrm{~kg}$ to calculate the body mass index (BMI, $\mathrm{kg} / \mathrm{m}^{2}$ ).

Screening blood pressure: After 5 minutes of rest, using a stethoscope and a mercury sphygmomanometer (auscultatory method), duplicate BP readings were taken 5 minutes apart and measurements averaged to obtain screening SBP and DBP.

Blood lipids: Total cholesterol (T-C) and high-density lipoprotein cholesterol (HDLC) levels were measured in serum with the Konelab 20i (Thermo Fisher Scientific, Vantaa, Finland). For statistical analysis, we computed the T-C/HDL-C ratio.

Hemoglobin A1c: HbA1c levels were determined by a turbidometric inhibition 
immunoassay method from EDTA plasma (Integra 400, Roche, Basel, Switzerland).

Renal function: We used the Modification of Diet in Renal Disease (MDRD) Study equation to estimate glomerular filtration rate (eGFR) from creatinine levels, age, sex, and ethnicity (36). Creatinine was measured in serum using an enzymatic colorimetric test (Cobas Integra 400 plus, Roche, Basel, Switzerland).

\section{Health behaviors}

Smoking: Participants who indicated that they currently smoked and/or had smoked at least one cigarette per day during one year in the past were categorized as ever smokers.

Alcohol consumption: Serum levels of $\gamma$-GT activity were used as a marker of alcohol abuse (37) and measured with an enzymatic colorimetric assay (Cobas Integra 400 plus).

Physical activity: We used the Actical ${ }^{\circledR}$ accelerometers (Montréal, Québec) to quantify and index physical activity with 3 (vigorous intensity), 2 (moderate intensity) or 1 (light intensity) (38).

\section{Coagulation measures}

Blood processing: Citrated blood samples were centrifuged at 1,500 rpm (2000g) for 15 minutes at room temperature. Citrated plasma samples were aliquoted and frozen at $80^{\circ} \mathrm{C}$ until analysis. Fibrinogen and D-dimer were determined by an accredited laboratory.

Von Willebrand factor: Plasma VWF:Ag (\%) were measured with a "sandwich" ELISA assay. A polyclonal rabbit anti-VWF antibody and a rabbit anti -VWF-HRP antibody (DAKO, South Africa) were used to form the assay. The 6th International Standard for VWF/FVIII was used to set the standard curve against which the samples were measured (39).

Fibrinogen: Plasma fibrinogen levels (g/l) were determined using a viscosity-based method (STA Compact, STAGO Diagnostic, Roche, France).

D-dimer: Plasma D-dimer levels (ng/ml) were determined with an immuno-based 
method (STA Compact, STAGO Diagnostic, Roche, France). There were respectively $47.6 \%$ and $39.7 \%$ of D-dimer values at rest and after stress below the limit of detection $(<220$ $\mathrm{ng} / \mathrm{ml})$. Undetectable values were substituted using the maximum likelihood method that generates values from the lognormal distribution of uncensored data under the assumption that censored values follow a lognormal distribution.

\section{Psychological questionnaires}

Depressive symptoms: We used the 9-item Patient Health Questionnaire (PHQ-9) to measure the frequency of depressive symptoms during the prior two weeks corresponding to criteria in the Diagnostic and Statistical Manual of Mental Disorders, Fourth Edition (40). Each item is rated on a 4-point scale $(0=$ "not at all", $1=$ "several days", $2=$ "more than half the days", 3="nearly every day") giving a possible global score of 0-27. PHQ-9 scores $\geq 10$ have $88 \%$ sensitivity and specificity for major depression (40). In our sample, Cronbach's $\alpha$ was 0.82 for Africans and 0.84 for Caucasians for the total scale.

Psychological distress: With the 28-item General Health Questionnaire (GHQ-28), we quantified perceived psychological distress in general related to symptoms of depression, anxiety, somatic complaints and social withdrawal over the past few weeks applying the binary scoring method. The global score provides a severity measure of total psychological distress. If exceeding the threshold of 4 , subjects are classified as achieving ,psychiatric caseness" (41). In our sample, Cronbach's $\alpha$ for the total scale was 0.91 for Africans and 0.90 for Caucasians.

\section{Statistical analysis}

We used SPSS version 21.0 for Windows to analyze the data with significance level at $\mathrm{p}<0.05$ (2-tailed). In case of a non-normal distribution, dependent variables were log transformed; for clarity, all data are given in original units. Chi-square test and Student's t-test 
with Levene's correction in the case of unequal variances were applied for data comparison between groups. We used multivariate analysis of variance (MANOVA) and of covariance (MANCOVA) to test for a significant association of ethnicity, PHQ-9 measures, GHQ-28 measures, and cardiovascular reactivity measures with coagulation measures. We only deemed results of individual coagulation measures to be significant if the MAN(C)OVA test statistic was significant, as this procedure protects against inflated Type I errors due to multiple tests of (likely) correlated dependent variables. Covariates were selected a priori based on the literature about demographic, cardiometabolic and life style factors affecting circulating levels of coagulation measures (42-44). Effect sizes are expressed as partial eta squared $\left(\eta_{\mathrm{p}}{ }^{2}\right)$ or partial correlation coefficients.

\section{RESULTS}

\section{Participant characteristics}

Table 1 shows the demographic and health characteristics of the 267 study participants per ethnic group. The percentage of men was higher in Africans than in Caucasians. In terms of cardiometabolic risk factors, Africans showed higher BMI, HbA1c, and screening BP despite using more antihypertensives - than Caucasians, while Caucasians had a higher TC/HDL-C ratio than Africans. Of the 20 subjects who took antihypertensive medications, 9 had thiazide diuretics, 6 had angiotensin-converting enzyme (ACE) inhibitors, 2 had angiotensin receptor blockers (ARB), and 3 had combination therapy (i.e., thiazide diuretic plus ACE inhibitor or ARB). Regarding health behaviors, Africans were physically less active and had higher $\gamma$-GT indicating more alcohol abuse than Caucasians. Africans were significantly more depressed and they also perceived more distress than Caucasians. 


\section{Coagulation measures at rest}

Ethnicity: Both MANOVA $\left(\mathrm{F}_{3,263}=43.28, \eta_{\mathrm{p}}{ }^{2}=0.330, \mathrm{p}<0.001\right)$ and MANCOVA $\left(\mathrm{F}_{3,252}=36.31, \eta_{\mathrm{p}}{ }^{2}=0.302, \mathrm{p}<0.001\right)$ showed a significant association between ethnicity and resting levels of the three coagulation measures. Table 2 shows that compared with Caucasians, Africans had significantly higher resting levels of VWF, fibrinogen, and D-dimer in the unadjusted as well as in the adjusted analyses.

Psychological questionnaires: To test for an association of PHQ-9 and GHQ-28 measures with resting levels of coagulation measures, we reran the above MANCOVA with continuous and categorical PHQ-9 and GHQ-28 measures as additional covariates (four separate models). Ethnicity remained associated with coagulation measures in all of these models (all p-values $<0.001$ ). However, coagulation measures showed no significant association with continuous PHQ-9 scores ( $\mathrm{p}=0.46)$, categorical PHQ-9 scores ( $\mathrm{p}=0.071)$, continuous GHQ-28 scores ( $\mathrm{p}=0.59)$, and GHQ-28 caseness $(\mathrm{p}=0.47)$.

Interaction between ethnicity and psychological questionnaires: Taking into account main effects of ethnicity and PHQ-9 and GHQ-28 scores, the interaction terms between ethnicity and the continuous as well as categorical PHQ-9 and GHQ-28 scores were not significant (all p-values $>0.10$ ).

\section{Stress-induced changes across all participants}

The mean level of perceived stress from the Stroop was 3.70 \pm 1.64 (range 0-7). Mental stress provoked significant responses in coagulation and hemodynamic measures. While VWF levels increased from $74.9 \pm 26.1 \%$ to $99.9 \pm 40.1 \%$ ( $p<0.001)$, fibrinogen levels decreased from $3.18 \pm 0.69 \mathrm{~g} / \mathrm{l}$ to $2.88 \pm 0.68 \mathrm{~g} / \mathrm{l}(\mathrm{p}<0.001)$. D-dimer levels showed no significant change $(320 \pm 339 \mathrm{ng} / \mathrm{ml}$ vs. $376 \pm 503 \mathrm{ng} / \mathrm{ml}, \mathrm{p}=0.44)$. Moreover, SBP $(134.4 \pm 16.2 \mathrm{mmHg}$ vs. 154.2 $\pm 20.8 \mathrm{mmHg}, \mathrm{p}<0.001), \mathrm{DBP}(78.6 \pm 9.3 \mathrm{mmHg}$ vs. $88.6 \pm 11.4 \mathrm{mmHg}, \mathrm{p}<0.001), \mathrm{HR}$ (66.8 \pm 10.7 bpm vs. $87.4 \pm 16.6$ bpm, $\mathrm{p}<0.001)$, and $\mathrm{CO}(6.59 \pm 1.81 \mathrm{l} / \mathrm{min}$ vs. $8.12 \pm 2.26 \mathrm{l} / \mathrm{min}$, 
$\mathrm{p}<0.001)$ all increased and SV $(99.6 \pm 23.9 \mathrm{ml}$ vs. $94.2 \pm 23.1 \mathrm{ml}, \mathrm{p}<0.001)$ and TPR $(1.02 \pm 0.46$ $\mathrm{mmHg} / \mathrm{ml} / \mathrm{s}$ vs. $0.99 \pm 0.51 \mathrm{mmHg} / \mathrm{ml} / \mathrm{s}, \mathrm{p}=0.013$ ) both decreased.

Association between hemodynamic and coagulation reactivity: Reactivity measures of VWF:Ag, fibrinogen, and D-dimer were not significantly correlated with each other (all pvalues $>0.25$ ). Separate MANOVA models for AUC measures of hemodynamic variables showed that reactivity in $\operatorname{DBP}\left(\mathrm{F}_{3,263}=3.83, \eta_{\mathrm{p}}{ }^{2}=0.042, \mathrm{p}=0.010\right), \mathrm{SV}\left(\mathrm{F}_{3,263}=3.35, \eta_{\mathrm{p}}{ }^{2}=0.037\right.$, $\mathrm{p}=0.020)$, and TPR $\left(\mathrm{F}_{3,263}=2.74, \eta_{\mathrm{p}}{ }^{2}=0.030, \mathrm{p}=0.044\right)$ were significantly associated with coagulation reactivity, whereas reactivity in $\operatorname{SBP}(p=0.44), \operatorname{HR}(p=0.83)$, and $\mathrm{CO}(\mathrm{p}=0.52)$ were not. Correlation analysis on individual measures revealed that VWF reactivity increased with greater SV reactivity $(r=0.18, p=0.003)$, but decreased with greater reactivity in DBP $(\mathrm{r}=-0.18, \mathrm{p}=0.003)$ and TPR $(\mathrm{r}=-0.14, \mathrm{p}=0.022)$. Fibrinogen and D-dimer reactivity were not significantly associated with DBP, SV, and TPR reactivity (all p-values $>0.12$ ).

\section{Stress-induced changes and ethnicity}

Perceived stress: Africans $(3.69 \pm 1.87)$ and Caucasians $(3.70 \pm 1.49)$ perceived the Stroop protocol as similarly stressful $(\mathrm{p}=0.94)$.

Coagulation reactivity: Using MANOVA $(\mathrm{p}=0.19)$ and MANCOVA $(\mathrm{p}=0.62)$ tests, there were no significant associations between ethnicity and poststress levels of coagulation measures (cf. Table 2 for unadjusted and adjusted poststress levels of VWF, fibrinogen, and D-dimer). However, there were significant association between ethnicity and coagulation reactivity in MANOVA $\left(\mathrm{F}_{3,263}=13.73, \eta_{\mathrm{p}}{ }^{2}=0.135, \mathrm{p}<0.001\right)$ and $\operatorname{MANCOVA}\left(\mathrm{F}_{3,251}=16.86\right.$, $\left.\eta_{\mathrm{p}}{ }^{2}=0.168, \mathrm{p}<0.001\right)$ tests. Table 1 shows that relative to Caucasians, Africans experienced lower procoagulant reactivity with less of an increase in VWF reactivity and more of a decrease in fibrinogen reactivity; D-dimer reactivity showed no significant difference between Africans and Caucasians. 
Hemodynamic reactivity: MANCOVA showed a significant association between ethnicity and AUC measures of hemodynamic variables $\left(\mathrm{F}_{6,248}=3.18, \eta_{\mathrm{p}}{ }^{2}=0.072, \mathrm{p}=0.005\right)$. Relative to Caucasians, Africans had lower SBP AUC (29.3 \pm 5.8 vs. $45.1 \pm 4.3, \eta_{\mathrm{p}}{ }^{2}=0.016$, $\mathrm{p}=0.043)$, and lower SV AUC $\left(-32.3 \pm 8.1\right.$ vs. $\left.5.4 \pm 6.1, \eta_{\mathrm{p}}{ }^{2}=0.049, \mathrm{p}<0.001\right)$, but greater TPR AUC $\left(0.40 \pm 0.27\right.$ vs. $\left.-0.30 \pm 0.20, \eta_{\mathrm{p}}{ }^{2}=0.029, \mathrm{p}=0.007\right)$. Ethnicity was not related to AUC measures of DBP $(p=0.46), H R(p=0.12)$, and CO $(p>0.09)$. Ethnicity did not significantly interact with AUC measures of any cardiovascular parameter to determine coagulation reactivity (all p-values $>0.18$ ).

Psychological questionnaires: After controlling for ethnicity MANCOVA tests showed no significant associations of continuous PHQ-9 scores ( $\mathrm{p}=0.71)$, categorical PHQ-9 scores $(\mathrm{p}=0.94)$, continuous GHQ-28 scores $(\mathrm{p}=0.18)$, and GHQ-28 caseness $(\mathrm{p}=0.91)$ with coagulation reactivity. However, there were significant interactions of ethnicity with continuous PHQ-9 scores $\left(\mathrm{F}_{3,249}=3.03, \eta_{\mathrm{p}}{ }^{2}=0.035, \mathrm{p}=0.030\right)$ and categorical continuous PHQ9 scores $\left(\mathrm{F}_{3,249}=3.80, \eta_{\mathrm{p}}{ }^{2}=0.044, \mathrm{p}=0.011\right)$, but not with continuous GHQ-28 scores $(\mathrm{p}=0.24)$ and GHQ-28 caseness $(\mathrm{p}=0.62)$.

Between-subject analysis revealed significant associations between ethnicity and continuous PHQ-9 scores for reactivity of VWF $\left(\eta_{\mathrm{p}}{ }^{2}=0.016, \mathrm{p}=0.044\right)$ and fibrinogen $\left(\eta_{\mathrm{p}}^{2}=0.020, \mathrm{p}=0.026\right)$, but not for $\mathrm{D}$-dimer reactivity $(\mathrm{p}=0.54)$. Partial correlation coefficients differed significantly between ethnic groups for VWF reactivity $(\mathrm{p}=0.029)$ and for fibrinogen reactivity $(\mathrm{p}=0.030)$. With greater continuously scaled PHQ-9 scores, indicating greater severity of depressive symptoms, VWF increased more in Africans $(r=0.202)$ than in Caucasians $(r=-0.074)$, whereas fibrinogen decreased more in Africans $(r=-0.215)$ than in Caucasians $(\mathrm{r}=0.058)$.

Similarly, ethnicity interacted with categorical PHQ-9 scores in determining VWF reactivity $\left(\eta_{\mathrm{p}}{ }^{2}=0.023, \mathrm{p}=0.015\right)$ and fibrinogen reactivity $\left(\eta_{\mathrm{p}}{ }^{2}=0.018, \mathrm{p}=0.035\right)$, but not $\mathrm{D}$ dimer reactivity $(\mathrm{p}=0.28)$. Figure 1 illustrates these interactions. Partial correlation 
coefficients significantly differed between groups for VWF reactivity $(\mathrm{p}=0.035)$ and for fibrinogen reactivity $(\mathrm{p}=0.047)$. PHQ-9 scores $\geq 10$ were directly correlated with VWF reactivity in Africans ( $\mathrm{r}=0.149)$, but inversely so in Caucasians ( $\mathrm{r}=-0.119)$. Moreover, PHQ-9 scores $\geq 10$ were inversely correlated with fibrinogen reactivity in Africans ( $r=-0.187)$, but directly so in Caucasians ( $\mathrm{r}=0.064)$.

We performed six complementary univariate analyses of covariance to test whether sympathetic activity as indicated by hemodynamic reactivity might relate to the direct association between continuous PHQ-9 scores and VWF reactivity in Africans versus Caucasians. However, all six three-way-interactions between ethnicity, continuous PHQ-9 scores and AUC measures of any hemodynamic parameter were non-significant (all pvalues $\geq 0.12$ ).

\section{DISCUSSION}

The main finding from our study is that Africans showed lower procoagulant reactivity in response to standardized acute laboratory mental stress when compared with Caucasians, controlling for a range of potentially confounding variables. Relative to Caucasians, VWF levels had increased less and fibrinogen levels had decreased more in Africans after stress relative to resting levels. One explanation for the diminished increase in VWF in Africans could be a "ceiling effect" as Africans started out with higher resting levels of VWF than Caucasians. For instance, previous studies in apparently healthy individuals reported stress-induced increases of VWF of 6\%, 9\%, and 30\% from resting levels which were respectively $91 \%, 98 \%$, and $95 \%$, in these studies $(4,45,46)$. This amount of an increase in VWF is clearly lower than in our Caucasian subjects who showed an increase in VWF of $60 \%$ starting out from average resting values of $64 \%$, but similar to our African subjects who showed an increase in VWF of $18 \%$ from average resting levels of $93 \%$. In contrast, a "floor effect" in Caucasians who started out on relatively lower resting fibrinogen 
levels might account for the greater decrease in poststress fibrinogen levels relative to resting levels in Africans. Another explanation for the blunted VWF reactivity in Africans might be their alpha-adrenergic vascular stress response, as was suggested by their increased TPR reactivity (22). Across all participants, lower VWF reactivity correlated with both greater TPR reactivity and lower SV reactivity, the latter likely reflecting increased afterload due to elevated TPR (22). Moreover, in Caucasians, the systemic vasodilation response during the Stroop test is largely mediated by the beta2-adrenergic receptor (47) whose stimulation through stress hormones will result in endothelial release of VWF into the circulation (19-21). A decrease in fibrinogen levels between rest and 10 minutes after the Stroop was seen across all study participants, and to an even greater extent in Africans than Caucasians. This is contrary to expectations, as fibrinogen levels were shown to increase in response to acute mental stress in several previous studies (2-4). The Stroop protocol provoked an average level of psychological distress that compares to similar protocols (10). However, previous studies suggest that the Stroop alone might be less effective in provoking a significant fibrinogen response (48) than combinations of the Stroop with other stressors (e.g., mental arithmetic) (45) or speech stressors (4). As there was no correlation of VWF reactivity with both fibrinogen and D-dimer reactivity in the present study, but in a previous one (49), it is also possible that the 1-minute stressor was too short to evoke a significant fibrinogen increase with fibrin formation and degradation further downstream. Nevertheless, previous studies showed coagulation and fibrinolysis activation within 2 to 5 minutes of acute mental stress $(50,51)$ and infusion with the stress hormone epinephrine (52). D-dimer did not increase in a previous study that combined the Stroop with mental arithmetic (53), but in studies that applied speech stressors to inflict social evaluative threat $(5,9)$. The sensitivity of the various D-dimer assays used in previous stress studies might also explain heterogeneous results (54). Saying that, previous stress reactivity studies also used different methods to measure fibrinogen (e.g., the functional Clauss method) (4) and VWF (e.g., enzyme-linked 
immunosorbent assays) (46). To our knowledge, a direct comparison of the sensitivity between different assays to detect reactivity in prothrombotic measures has not been performed, but would seem important to reconcile heterogeneous study findings.

Coagulation reactivity was specifically associated with depressive symptoms (i.e., PHQ-9 scores) as opposed to more general psychological distress (i.e., GHQ-28 scores). More severe depressive symptoms were associated with greater VWF reactivity in Africans compared with Caucasians. This concurs with the notion that depressive mood along a continuum of severity is associated with an increased risk of incident CVD and recurrent cardiac events (55). However, poststress levels of VWF and fibrinogen were similar in Africans and Caucasians, fibrinogen decreased more in depressed Africans than depressed Caucasians, and VWF reactivity was, on the whole, lower in Africans than in Caucasians; therefore, a depression-associated increase in CVD risk in Africans through a pathway of stress-induced VWF increase would need to be shown in prospective studies.

Ethnical differences in coagulation are still poorly understood with studies showing enhanced clotting but also increased bleeding tendency in various clinical settings in Africans compared with Caucasians (56). We found resting levels of VWF:Ag, fibrinogen, and Ddimer to be higher in Africans than Caucasians, which is in agreement with some previous studies (57-59). Theoretically, this potential predisposition to hypercoagulability might contribute to the CVD risk in Africans. In contrast, lower increase in VWF levels and greater decrease in fibrinogen levels both with acute mental stress could be viewed a hypoactive fight-flight response (60). As opposed to constitutively released VWF, the VWF released from the endothelium after stimulation consists of large and hemostatically highly active multimers (61). Moreover, although not investigated in our study, acute stress also activates fibrinolysis $(2,45,50)$. Therefore, the inability to mount physiologic hypercoagulability might subject Africans to an increased risk of bleeding in fight-flight situations either upon injury or even spontaneously. Therefore, the almost absent VWF response in the non-depressed 
Africans might be as harmful as the increased VWF reactivity in the depressed Africans for the maintenance of the haemostatic balance between thrombosis and haemorrhage during acute mental stress.

Rigorous selection of participants on medical characteristics to minimize confounding of coagulation measures was a strength of our study, but also might reduce generalizability of study findings to the larger African population and those with established CVD. We selected three coagulation measures that were previously shown to be stress-responsive. However, they do not cover the entire dynamics of the coagulation and fibrinolysis pathways during acute mental stress. A speech stressor inflicting social evaluative threat and measuring coagulation measures longer into the recovery period from stress might have provided additional informative data. The Stroop test provoked substantial increases in BP and HR, but there is an ongoing debate as to whether cardiovascular reactivity data gained from standardized lab stressor are generalizable to real life situations (62). We controlled our analysis for antihypertensive medications as a group but due to insufficient statistical power could not take into account potential class effects of the different antihypertensives on outcomes. We did not include a non-stress control group. Therefore we were unable to account for circadian changes in coagulation measures, particularly so a decrease in plasma fibrinogen levels during the morning hours. We are unable to account for the possibility that stress-related changes in clearance impacted plasma concentrations of coagulation measures. For instance, glycosylation of VWF influences its clearance by the liver, which, moreover, occurs more rapidly in individuals with blood group $\mathrm{O}$ than in non-O individuals (63); unfortunately, information on $\mathrm{ABO}$ blood group was not available in our study. The difference in liver function between Africans and Caucasians seems not an apparent explanation for the ethnic difference in VWF reactivity because the relation between ethnicity and procoagulant reactivity persisted after adjustment for $\gamma$-GT activity. 
Taken together, Africans seem to have lower procoagulant reactivity with acute mental stress than Caucasians. This observation might partially be explained by ethnic differences in the vascular stress response. Depressive symptoms may modulate coagulation reactivity against an ethnic background. The potential clinical implications of our findings for CVD risk and bleeding disorders in Africans need further studies.

\section{ACKNOWLEDGEMENTS}

The authors are grateful to Péter Szabolcs, Chrissie Lessing, and Tina Scholtz for their technical assistance and support. The study was partly funded by The Metabolic Syndrome Institute, France; the Medical Research Council, National Research Foundation, North-West University, and North-West Department of Education, South Africa.

\section{REFERENCES}

1. Borissoff JI, Spronk HM, ten Cate H. The hemostatic system as a modulator of atherosclerosis. N Engl J Med 2011; 364: 1746-1760.

2. von Känel R, Mills PJ, Fainman C, et al. Effects of psychological stress and psychiatric disorders on blood coagulation and fibrinolysis: a biobehavioral pathway to coronary artery disease? Psychosom Med 2001; 63: 531-544.

3. Thrall G, Lane D, Carroll D, et al. A systematic review of the effects of acute psychological stress and physical activity on haemorheology, coagulation, fibrinolysis and platelet reactivity: Implications for the pathogenesis of acute coronary syndromes. Thromb Res 2007; 120: 819-847.

4. von Känel R, Preckel D, Zgraggen L, et al. The effect of natural habituation on coagulation responses to acute mental stress and recovery in men. Thromb Haemost 2004; 92: 1327-1335. 
5. Wirtz PH, Ehlert U, Emini L, et al. Anticipatory cognitive stress appraisal and the acute procoagulant stress response in men. Psychosom Med 2006; 68: 851-858.

6. Ellins E, Halcox J, Donald A, et al. Arterial stiffness and inflammatory response to psychophysiological stress. Brain Behav Immun 2008; 22: 941-948.

7. Brydon L, Steptoe A. Stress-induced increases in interleukin-6 and fibrinogen predict ambulatory blood pressure at 3-year follow-up. J Hypertens 2005; 23: 1001-1007.

8. Strike PC, Magid K, Brydon L, et al. Exaggerated platelet and hemodynamic reactivity to mental stress in men with coronary artery disease. Psychosom Med 2004; 66: 492-500.

9. von Känel R, Dimsdale JE, Ziegler MG, et al. Effect of acute psychological stress on the hypercoagulable state in subjects (spousal caregivers of patients with Alzheimer's disease) with coronary or cerebrovascular disease and/or systemic hypertension. Am J Cardiol 2001; 87: 1405-1408.

10. Strike PC, Magid K, Whitehead DL, et al. Pathophysiological processes underlying emotional triggering of acute cardiac events. Proc Natl Acad Sci U S A 2006; 103: 43224327.

11. Aschbacher K, Roepke SK, von Känel R, et al. Persistent versus transient depressive symptoms in relation to platelet hyperactivation: a longitudinal analysis of dementia caregivers. J Affect Disord 2009; 116: 80-87.

12. von Känel R, Dimsdale JE, Adler KA, et al. Effects of depressive symptoms and anxiety on hemostatic responses to acute mental stress and recovery in the elderly. Psychiatry Res 2004; 126: 253-264.

13. Lippi G, Montagnana M, Favaloro EJ, et al. Mental depression and cardiovascular disease: a multifaceted, bidirectional association. Semin Thromb Hemost 2009; 35: 325336. 
14. Sliwa K, Wilkinson D, Hansen C, et al. Spectrum of heart disease and risk factors in a black urban population in South Africa (the Heart of Soweto Study): a cohort study. Lancet 2008; 371: 915-922.

15. Pieters M, de Maat MP, Jerling JC, et al. Fibrinogen concentration and its role in CVD risk in black South Africans-effect of urbanisation. Thromb Haemost 2011; 106: 448-456.

16. Hamer M, Malan L, Schutte AE, et al. Conventional and behavioral risk factors explain differences in sub-clinical vascular disease between black and Caucasian South Africans: the SABPA study. Atherosclerosis 2011; 215: 237-242.

17. Hamer M, Malan NT, Harvey BH, et al. Depressive symptoms and sub-clinical atherosclerosis in Africans: role of metabolic syndrome, inflammation and sympathoadrenal function. Physiol Behav 2011; 104: 744-748.

18. Preckel D, von Känel R. Regulation of hemostasis by the sympathetic nervous system: any contribution to coronary artery disease? Heartdrug 2004; 4: 123-130.

19. von Känel R, Dimsdale JE. Effects of sympathetic activation by adrenergic infusions on hemostasis in vivo. Eur J Haematol 2000; 65: 357-369.

20. von Känel R, Dimsdale JE, Adler KA, et al. Effects of nonspecific beta-adrenergic stimulation and blockade on blood coagulation in hypertension. J Appl Physiol 2003; 94: 1455-1459.

21. von Känel R, Kudielka BM, Helfricht S, et al. The effects of aspirin and nonselective beta blockade on the acute prothrombotic response to psychosocial stress in apparently healthy subjects. J Cardiovasc Pharmacol 2008; 51: 231-238.

22. Huisman HW, Schutte AE, Schutte R, et al. Exploring the link between cardiovascular reactivity and end-organ damage in African and Caucasian men: the SABPA study. Am J Hypertens 2013; 26: 68-75.

23. World Medical Association. Declaration of Helsinki. Ethical principles of medical research involving human subjects. J Indian Med Assoc 2009; 107: 403-405. 
24. Lip GY, Lowe GD, Rumley A, et al. Increased markers of thrombogenesis in chronicatrial fibrillation: effects of warfarin treatment. Br Heart J 1995; 73: 527-533.

25. Baker J, Ayenew W, Quick H, et al. High-density lipoprotein particles and markers of inflammation and thrombotic activity in patients with untreated HIV infection. J Infect Dis 2010; 201: 285-292.

26. Brotman DJ, Girod JP, Posch A, et al. Effects of short-term glucocorticoids on hemostatic factors in healthy volunteers. Thromb Res 2006; 118: 247-252.

27. Prasad RN, Koh SC, Viegas OA, et al. Effects on hemostasis after two-year use of low dose combined oral contraceptives with gestodene or levonorgestrel. Clin Appl Thromb Hemost 1999; 5: 60-70.

28. Gebara OC, Jimenez AH, McKenna C, et al. Stress-induced hemodynamic and hemostatic changes in patients with systemic hypertension: effect of verapamil. Clin Cardiol 1996; 19: $205-211$.

29. Geiser F, Conrad R, Imbierowicz K, et al. Coagulation activation and fibrinolysis impairment are reduced in patients with anxiety and depression when medicated with serotonergic antidepressants. Psychiatry Clin Neurosci 2011; 65: 518-525.

30. Falaschi P, Proietti A, De Angelis C, et al. Effects of mental stress on cardiovascular and endocrine response in Air Force Academy cadets. Neuro Endocrinol Lett 2003; 24: 197 202.

31. Imholz BP, Wieling W, van Montfrans GA, et al. Fifteen years experience with finger arterial pressure monitoring: assessment of the technology. Cardiovasc Res 1998; 38: 605616.

32. Wesseling KH, Jansen LR, Settels JJ, Schreuder JJ. Computation of aortic flow from pressure in humans using a nonlinear, three-element model. J Appl Physiol 1993; 74: 2566-2573. 
33. Harms MP, Wesseling KH, Pott F, et al. Continuous stroke volume monitoring by modelling flow from non-invasive measurement of arterial pressure in humans under orthostatic stress. Clin Sci 1999; 97: 291-301.

34. Jansen JR, Schreuder JJ, Mulier JP, Smith NT, Settels JJ, Wesseling KH. A comparison of cardiac output derived from the arterial pressure wave against thermodilution in cardiac surgery patients. Br J Anaesth 2001; 87: 212-222.

35. Pruessner JC, Kirschbaum C, Meinlschmid G, et al. Two formulas for computation of the area under the curve represent measures of total hormone concentration versus time dependent change. Psychoneuroendocrinology 2003; 28: 916-931.

36. Levey AS, Bosch JP, Lewis JB, et al. A more accurate method to estimate glomerular filtration rate from serum creatinine: a new prediction equation. Modification of Diet in Renal Disease Study Group. Ann Intern Med 1999; 130: 461-470.

37. Sharpe PC. Biochemical detection and monitoring of alcohol abuse and abstinence. Ann Clin Biochem 2001; 38: 652-664.

38. Heil DP. Predicting activity energy expenditure using the Actical ${ }^{\circledR}$ Activity Monitor. Res Q Exercise Sport 2006; 77: 64-80.

39. Meiring M, Badenhorst PN, Kelderman M. Performance and utility of a cost-effective collagen-binding assay for the laboratory diagnosis of Von Willebrand disease. Clin Chem Lab Med 2007; 45: 1068-1072.

40. Kroenke K, Spitzer RL, Williams JB. The PHQ-9: validity of a brief depression severity measure. J Gen Intern Med 2001; 16: 606-613.

41. Goldberg DP, Hillier VF. A scaled version of the General Health Questionnaire. Psychol Med 1979; 9: 139-145.

42. Fibrinogen Studies Collaboration, Kaptoge S, White IR, Thompson SG, et al. Associations of plasma fibrinogen levels with established cardiovascular disease risk factors, inflammatory markers, and other characteristics: individual participant meta 
analysis of 154,211 adults in 31 prospective studies: the fibrinogen studies collaboration. Am J Epidemiol 2007; 166: 867-879.

43. Lee KW, Lip GY. Effects of lifestyle on hemostasis, fibrinolysis, and platelet reactivity: a systematic review. Arch Intern Med 2003; 163: 2368-2392.

44. Dubin R, Cushman M, Folsom AR, et al. Kidney function and multiple hemostatic markers: cross sectional associations in the multi-ethnic study of atherosclerosis. BMC Nephrol 2011; 12: 3.

45. Jern C, Eriksson E, Tengborn L, et al. Changes of plasma coagulation and fibrinolysis in response to mental stress. Thromb Haemost 1989; 62: 767-771.

46. Hamer M, Gibson EL, Vuononvirta R, Williams E, Steptoe A. Inflammatory and hemostatic responses to repeated mental stress: individual stability and habituation over time. Brain Behav Immun 2006; 20: 456-569.

47. Freyschuss U, Hjemdahl P, Juhlin-Dannfelt A, et al. Cardiovascular and sympathoadrenal responses to mental stress: influence of beta-blockade. Am J Physiol 1988; 255: H1443H1451.

48. Muldoon MF, Herbert TB, Patterson SM, et al. Effects of acute psychological stress on serum lipid levels, hemoconcentration, and blood viscosity. Arch Intern Med 1995; 155 : 615-620.

49. Zgraggen L, Fischer JE, Mischler K, et al. Relationship between hemoconcentration and blood coagulation responses to acute mental stress. Thromb Res 2005; 115: 175-183.

50. Palermo A, Bertalero P, Pizza N, Amelotti R, Libretti A. Decreased fibrinolytic response to adrenergic stimulation in hypertensive patients. J Hypertens Suppl 1989; 7: S162-S163.

51. de Boer D, Ring C, Wood M, Ford C, Jessney N, McIntyre D, Carroll D. Time course and mechanisms of mental stress-induced changes and their recovery: hematocrit, colloid osmotic pressure, whole blood viscosity, coagulation times, and hemodynamic activity. Psychophysiology 2007; 44: 639-649. 
52. Cash JD, Lind AR, McNicol GW, Woodfield DG. Fibrinolytic and forearm blood flow responses to intravenous adrenaline in healthy subjects. Lif Sci 1969; 8: 207-213.

53. Steptoe A, Kunz-Ebrecht S, Rumley A, et al. Prolonged elevations in haemostatic and rheological responses following psychological stress in low socioeconomic status men and women. Thromb Haemost 2003; 89: 83-90.

54. Righini M, Perrier A, De Moerloose P, et al. D-Dimer for venous thromboembolism diagnosis: 20 years later. J Thromb Haemost 2008; 6: 1059-1071.

55. Frasure-Smith N, Lespérance F. Depression and cardiac risk: present status and future directions. Heart 2010; 96: 173-176.

56. Mayr FB, Spiel AO, Leitner JM, et al. Racial differences in endotoxin-induced tissue factor-triggered coagulation. J Thromb Haemost 2009; 7: 634-640.

57. Lutsey PL, Cushman M, Steffen LM, et al. Plasma hemostatic factors and endothelial markers in four racial/ethnic groups: the MESA study. J Thromb Haemost 2006; 4: 26292635.

58. Gader A, Bahakim H, Awadalla S, et al. Ethnic variations in the haemostatic system: comparison between Arabs, Westerners (Europeans and Americans), Asians and Africans. Blood Coagul Fibrinolysis 1995; 6: 537-542.

59. Sukhu K, Poovalingam V, Mahomed R, et al. Ethnic variation in von Willebrand factor levels can influence the diagnosis of von Willebrand disease. Clin Lab Haematol 2003; 25: 247-249.

60. Lovallo WR. Do low levels of stress reactivity signal poor states of health? Biol Psychol 2011; 86: 121-128.

61. Sporn LA, Marder VJ, Wagner DD. Inducible secretion of large, biologically potent von Willebrand factor multimers. Cell 1986; 46: 185-190.

62. Kamarck TW, Lovallo WR. Cardiovascular reactivity to psychological challenge: conceptual and measurement considerations. Psychosom Med 2003; 65: 9-21. 
63. Lenting PJ, Van Schooten CJ, Denis CV. Clearance mechanisms of von Willebrand factor and factor VIII. J Thromb Haemost 2007; 7: 1753-1760. 


\section{Legend to Figure 1}

The figure depicts the multivariate-adjusted interaction effects between ethnicity and categorical depression (i.e., score $\geq 10$ on the PSQ-9 total depressive symptom scale) on von Willebrand factor (VWF) and fibrinogen reactivity (i.e., relative change in levels of coagulation measures in $\%$ from resting levels to 10 minutes after the Stroop test). Relative to non-depressed individuals (Dep -) of the respective ethnicity, VWF reactivity was significantly greater in Africans vs. Caucasians with depression (Dep +). Fibrinogen reactivity showed a significantly greater decrease in depressed Africans than in depressed Caucasians (cf. text for detailed statistics). Africans/Dep + $(n=47)$, Africans/Dep - $(n=55)$, Caucasians/Dep $+(n=29)$, Caucasians/Dep $-(n=136)$. 
Table 1. Characteristics of 267 study participants

\begin{tabular}{|l|l|l|l|}
\hline Variable & $\begin{array}{l}\text { Africans } \\
(\mathbf{n = 1 0 2})\end{array}$ & $\begin{array}{l}\text { Caucasians } \\
(\mathbf{n = 1 6 5})\end{array}$ & P-value \\
\hline Gender (\% men) & 62.7 & 49.7 & 0.037 \\
\hline Age (yrs) & $43.4 \pm 8.2$ & $44.1 \pm 11.0$ & 0.580 \\
\hline Body mass index (kg/m $\left.{ }^{2}\right)$ & $29.9 \pm 7.8$ & $27.6 \pm 5.6$ & 0.008 \\
\hline Total cholesterol (mmol/l) & $4.76 \pm 1.17$ & $5.50 \pm 1.22$ & $<0.001$ \\
\hline HDL-cholesterol (mmol/l) & $1.15 \pm 0.36$ & $1.20 \pm 0.41$ & 0.339 \\
\hline Total cholesterol/HDL-cholesterol ratio & $4.51 \pm 1.86$ & $4.98 \pm 1.60$ & 0.028 \\
\hline Systolic blood pressure (mmHg) & $134.4 \pm 19.6$ & $126.7 \pm 13.5$ & $<0.001$ \\
\hline Diastolic blood pressure (mmHg) & $88.4 \pm 12.6$ & $83.1 \pm 9.6$ & $<0.001$ \\
\hline Hemoglobin A1c (\%) & $6.05 \pm 1.12$ & $5.48 \pm 0.41$ & $<0.001$ \\
\hline Creatinine ( $\mu$ mol/l) & $77.6 \pm 13.5$ & $74.2 \pm 15.3$ & 0.069 \\
\hline Estimated GFR (ml/min/1.73m $\left.{ }^{2}\right)$ & $114.7 \pm 29.3$ & $112.0 \pm 27.2$ & 0.453 \\
\hline Ever smoker (\%) & 25.5 & 21.2 & 0.419 \\
\hline Gamma glutamyl transferase (U/L) & $64.6 \pm 75.8$ & $26.9 \pm 36.2$ & $<0.001$ \\
\hline Physical activity index & $1.33 \pm 0.57$ & $1.62 \pm 0.70$ & 0.001 \\
\hline Antihypertensive drugs (\%) & 12.7 & 4.2 & 0.010 \\
\hline Patient Health Questionnaire-9 (scores) & & & $<0.001$ \\
\hline Total depressive symptoms & $9.40 \pm 5.60$ & $5.70 \pm 4.73$ & $<001$ \\
\hline Moderate depression (cut-off $\geq 10)(\%)$ & 46.1 & 17.6 & $<0.001$ \\
\hline General Health Questionnaire-28 (scores) & & $3.79 \pm 4.80$ & $<.001$ \\
\hline Total psychological distress & $8.50 \pm 6.71$ & 33.3 & \\
\hline Cases (cut-off $>4)(\%)$ & 64.7 & & $<$ \\
\hline & & & \\
\hline
\end{tabular}

Data are given as means \pm SD or percentage values. P-value refers to group differences.

GFR, glomerular filtration rate; HDL, high-density lipoprotein 
Table 2. Coagulation measurements per ethnic group at rest and after mental stress

\begin{tabular}{|c|c|c|c|c|c|c|c|c|c|c|c|c|c|}
\hline \multirow[t]{2}{*}{ Condition } & \multirow{2}{*}{$\begin{array}{r}\text { Adj. } \\
+\end{array}$} & \multicolumn{4}{|c|}{ von Willebrand factor } & \multicolumn{4}{|l|}{ Fibrinogen } & \multicolumn{4}{|l|}{ D-dimer } \\
\hline & & Africans & Caucasians & $\mathrm{ES}$ & $\mathrm{P}$ & Africans & Caucasians & $\mathrm{ES}$ & $\mathrm{P}$ & Africans & Caucasians & $\mathrm{ES}$ & $\mathrm{P}$ \\
\hline \multirow[t]{2}{*}{ Rest } & - & $93.2 \pm 28.4 \%$ & $63.6 \pm 16.5 \%$ & 0.330 & $<0.001$ & $3.35 \pm 0.82 \mathrm{~g} / 1$ & $3.07 \pm 0.57 \mathrm{~g} / 1$ & 0.030 & 0.004 & $354 \pm 340 \mathrm{ng} / \mathrm{ml}$ & $298 \pm 338 \mathrm{ng} / \mathrm{ml}$ & 0.015 & 0.044 \\
\hline & + & $94.2 \pm 2.4 \%$ & $62.9 \pm 1.8 \%$ & 0.291 & $<0.001$ & $3.33 \pm 0.06 \mathrm{~g} / 1$ & $3.09 \pm 0.05 \mathrm{~g} / 1$ & 0.029 & 0.006 & $379 \pm 37 \mathrm{ng} / \mathrm{ml}$ & $283 \pm 28 \mathrm{ng} / \mathrm{ml}$ & 0.030 & 0.005 \\
\hline \multirow[t]{2}{*}{ Poststress } & - & $101.8 \pm 34.1 \%$ & $98.7 \pm 43.4 \%$ & 0.010 & 0.101 & $2.95 \pm 0.71 \mathrm{~g} / 1$ & $2.84 \pm 0.65 \mathrm{~g} / 1$ & 0.006 & 0.208 & $398 \pm 515 \mathrm{ng} / \mathrm{ml}$ & $363 \pm 496 \mathrm{ng} / \mathrm{ml}$ & 0.004 & 0.302 \\
\hline & + & $97.5 \pm 4.4 \%$ & $101.4 \pm 3.3 \%$ & $<0.001$ & 0.817 & $2.91 \pm 0.07 \mathrm{~g} / 1$ & $2.86 \pm 0.05 \mathrm{~g} / 1$ & 0.001 & 0.593 & $426 \pm 56 \mathrm{ng} / \mathrm{ml}$ & $345 \pm 42 \mathrm{ng} / \mathrm{ml}$ & 0.006 & 0.217 \\
\hline \multirow[t]{2}{*}{ Reactivity } & - & $18.1 \pm 53.6 \%$ & $60.1 \pm 70.2 \%$ & 0.110 & $<0.001$ & $-11.2 \pm 11.6 \%$ & $-7.4 \pm 12.6 \%$ & 0.022 & 0.016 & $64.5 \pm 241.9 \%$ & $74.5 \pm 319.9 \%$ & 0.002 & 0.499 \\
\hline & + & $10.2 \pm 7.0 \%$ & $65.0 \pm 5.2 \%$ & 0.140 & $<0.001$ & $-11.7 \pm 1.4 \%$ & $-7.2 \pm 1.0 \%$ & 0.023 & 0.016 & $48.1 \pm 32.6 \%$ & $84.7 \pm 24.6 \%$ & 0.005 & 0.270 \\
\hline
\end{tabular}

Data are given as means \pm SD for unadjusted values (-) and as means \pm SEM for adjusted values (+) with P-values for group comparisons.

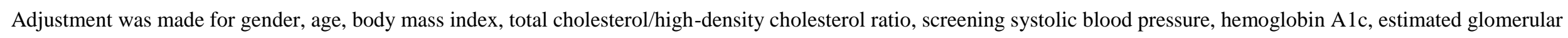
filtration rate, smoking, gamma glutamyl transferase, physical activity, and antihypertensive drugs for resting values and additionally for perceived stress with the Stroop test for post-stress and reactivity values. Reactivity measures are expressed as percentage change of posttstress values from resting values.

Adj., adjustment; ES, effect sizes (partial eta-squared). 


\section{Extra Table}

\section{What is known about this topic?}

- Acute mental stress induces a hypercoagulable state that may contribute to atherothrombotic diseases.

- Depressive symptoms are associated with the procoagulant stress response.

- There are ethnic differences in coagulation between Africans and Caucasians.

What this paper adds

- Africans show lower procoagulant reactivity in response to acute laboratory mental stress than Caucasians

- Explanation might be higher resting levels of coagulation factors in Africans than Caucasians and ethnic differences in the vascular stress response.

- Depressive symptoms are associated with greater stress reactivity of von Willebrand factor in Africans relative to Caucasians. 
Figure 1: Coagulation reactivity related to ethnicity and depression
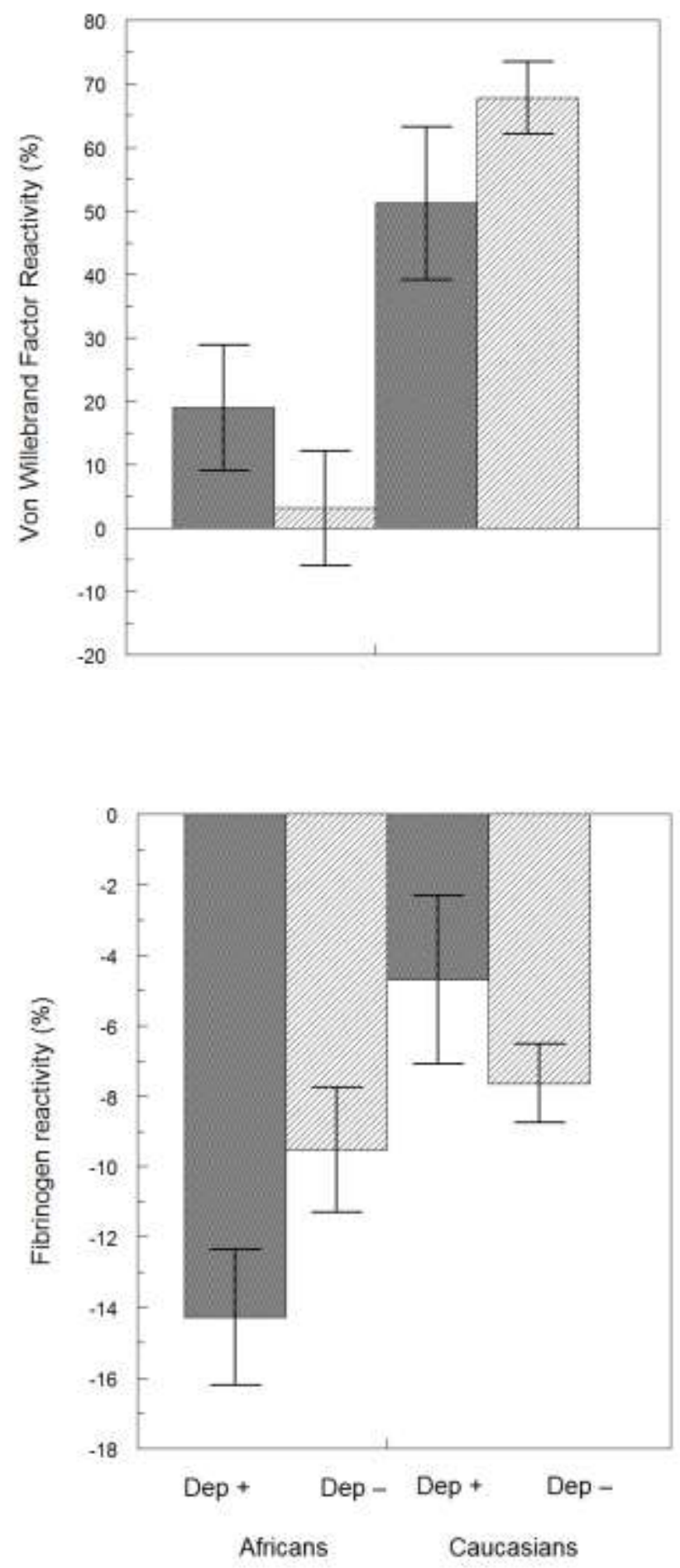